Célia Landmann Szwarcwald 1

Euclides Ayres de Casti lho 2

Aristides Barbosa Júnior 2

Maria Rebeca Otero Gomes 2

Eduardo A. M. Martins Costa 1

Bruno Vasques Maletta 1

Ricardo Fernando Mendelshon de Carval ho 3

Severino Ramos de Oliveira 3

Pedro Chequer 2

\section{Comportamento de risco dos conscritos do Exército Brasileiro, 1998: uma apreciação da infecção pelo HIV segundo diferenciais sócio-econômicos}

\author{
Socioeconomic differences in HIV risk \\ behavior among Brazilian Military conscripts
}

\footnotetext{
1 Departamento de Informações em Saúde, Centro de Informação Científica e Tecnológica, Fundação Oswaldo Cruz. Av. Brasil 4365, Rio de Janeiro, RJ 21045-900, Brasil.

2 Coordenação Nacional de DST e AIDS, Secretaria de Políticas de Saúde, Ministério da Saúde Esplanada dos Ministérios, Bloco G, Edifício Sede, Sobreloja, Brasília, DF 70058-900, Brasil.

3 Diretoria de Saúde do Exército Brasileiro. QGEx, Bloco G, 2o andar, SMU, Brasília, DF 70630-901, Brasil.
}

\begin{abstract}
A study of HIV-related risk behavior was carried out in 1998 among Brazilian military conscripts aged 17-20 years. A sample of 30,318 subjects was selected in three strata, pertaining to counties from: 1 ) the North and Central-West (N/CW); 2) South (S); and the states of Rio de Janeiro and São Paulo. HIV prevalence rates were estimated in all strata. The objective of this paper was to analyze the results according to differences in soci oeconomic status (SES). The statistical analysis used an index of sexual risk behavior and logistic regression models. The N/CW stratum showed the worst indicators for SES, sexual risk behavior, and sexually transmitted infections (STIS), as well as the highest HIV seroprevalence rate. The best indicators for all variables were found in the RJ/SP stratum. The South showed intermediate results. Level of schooling al so played a relevant role. In all three strata the conscripts with an incomplete high school education displayed the worst sexual risk behavior index, shown to bea relevant predictor of STI-related problems, including HIV infection.
\end{abstract}

Key words Acquired Immunodeficiency Syndrome; HIV Seroprevalence; Sexual Behavior; Military Personnel; Social Conditions

Resumo Uma pesquisa de comportamento de risco ao HIV foi conduzida entre conscritos (1720 anos) do Exército em 1998. A amostra (30.318) foi selecionada em três estratos: 1) municípios das regi ões Norte e Centro-Oeste (N/CO); 2) municípios da regi ão Sul (S); 3) municípios dos estados do Rio de Janeiro e São Paulo (RJ/SP), estimando-se a prevalência de infecção pel o HIV em cada estrato. O obj etivo deste trabalho foi o de apreciar os resultados por nível sóci o-econômico (NSE). A análi se estatística utilizou um índice de comportamento sexual de risco para HIV e model os de regressão logísti ca. O estrato N/CO apresentou os piores indicadores - os rel ativos ao NSE, ao comportamento sexual de risco ou aos problemas relaci onados às infecções sexual mente transmissíveis (IST) - assi m como a mai or proporção de infectados por HIV. Os mel hores indicadores foram encontrados no estrato RJ/SP. O Sul mostrou resul tados intermediários. O grau de escolaridade teve papel rel evante. Os conscritos com primei ro grau incompl eto apresentaram o pior índice de comportamento sexual de risco, o qual se mostrou importante preditor dos problemas rel acionados às IST, incluindo a infecção por HIV.

Palavras-chave Síndrome da I munodefi ciência Adquirida; Soroprevalência de HIV; Comportamento Sexual; Militares; Condições Sociais 
Introdução

Os adolescentes e adultos jovens constituem um grupo de risco crescente para as infecções sexualmente transmissíveis (IST), incluindo a infecção pelo HIV. A maior vulnerabilidade dos jovens decorre de falhas ou inconsistências no uso de preservativos (Anderson et al., 1990; Kann et al., 1998) em paralelo às elevadas taxas de atividade sexual com diferentes parceiros (Ku et al., 1993; Santelli et al., 1998).

A adolescência é etapa da vida marcada por complexo processo de desenvolvimento biológico, psíquico e social. É principalmente nesta fase que as influências contextuais, externas à família, tomam maior magnitude, pois vão implicar a tomada de decisões de condutas e contribuir para a definição de estilos de vida (Ruzani \& Szwarcwald, 1999). Neste período, o jovem se "arrisca", oscilando entre as situações de risco "calculado", decorrentes de ação pensada, e as de risco "insensato", nas quais, expondo-se gratuitamente, pode comprometer sua vida de forma irreversível (DiClemente et al., 1996).

Assim, com a expressão mais efetiva dos impulsos sexuais em função da maturação reprodutiva, a gravidez precoce e as IST são problemas cada vez mais relevantes nesta população (Warren et al., 1998). Nos Estados Unidos da América do Norte, estima-se que mais da metade da população infectada pelo HIV adquire a infecção entre as idades de 15 a 24 anos (Peersman \& Levy, 1998).

Inúmeros estudos mostram que jovens carentes ou residentes em comunidades de baixa renda estão sujeitos, com maior freqüência, aos comportamentos ditos de "risco" que influenciam a sua saúde (Sells \& Blum, 1996). No caso particular de problemas de saúde relacionados às IST, adolescentes das chamadas "minorias sociais" demonstram maior atividade sexual, idade mais precoce de início de relacionamento sexual, maior número de parceiros sexuais e uso menos freqüente de preservativo (Ford et al., 1994; Reitman et al., 1996). No Brasil, a Pesquisa Nacional sobre Demografia e Saúde (PNDS) revelou igual mente que jovens com baixo nível de instrução são os que demostram maior atividade sexual, iniciam a vida sexual mais cedo e apresentam o menor percentual de uso de preservativo masculino na última relação sexual (BEMFAM, 1997a).

A primeira investigação realizada com conscritos do Exército Brasileiro foi conduzida em 1992, a partir da amostra de jovens que se alistaram durante esse ano em Campo Grande, Mato Grosso do Sul (Souza, 1994). A partir de 1996, a Coordenação Nacional de DST e AIDS do Ministério da Saúde, em convênio com o Ministério do Exército do Brasil, tem realizado pesquisas por amostragem com conscritos, obtendo informações de jovens brasileiros, a maioria com 18 anos de idade e oriundos de todas as regiões do País e de estratos sociais diversos acerca de comportamento sexual e percepções relativas ao HIV/ AIDS (CNDST/AIDS, 1998a; 1998b).

A "Pesquisa Comportamental de Conscritos do Exército Brasil lei ro" - realizada por ocasião da apresentação dos conscritos no ano de 1998 - coletou informações a respeito das condições sócio-econômicas, práticas sexuais, uso de drogas injetáveis e problemas relacionados às IST, permitindo associá-las, pela primeira vez, aos resultados de exames sorológicos para a infecção pelo HIV. Este trabal ho tem o objetivo de analisar os dados desta pesquisa, apreciando os resultados à luz dos diferenciais sócioeconômicos.

\section{Metodologia}

Foram selecionados 30.318 conscritos, obedecendo a um plano de amostragem por estratificação em múltiplos estágios. Foram considerados três estratos: o primeiro, formado por municípios dos Estados de Rondônia, Amazonas, Pará, Tocantins e Mato Grosso (estrato N/CO); o segundo formado por municípios dos Estados do Paraná, Santa Catarina e Rio Grande do Sul (estrato S); e o terceiro formado por municípios dos Estados do Rio de Janeiro e São Paulo (estrato RJ/SP).

Os estratos foram selecionados de acordo com os resultados de estudo elaborado com os dados notificados de AIDS no período de 199095 (Szwarcwald et al., 1998). Os dois primeiros estratos foram escolhidos para a pesquisa em virtude de serem compostos por Estados que apresentaram grandes percentuais de crescimento relativo das taxas de incidência de AIDS do período 1990-1992 para o de 1993-1995. Verificando-se, por outro lado, que os Estados do Rio de Janeiro e São Paulo são os que demonstraram a maior estabilidade da epidemia, foram escolhidos como estrato-controle para comparação inter-regional dos resultados.

Em cada estrato, prosseguiu-se o processo de amostragem em dois estágios. Para todos os Estados enfocados, foram listados os aumentos relativos percentuais das taxas de incidência de AIDS do período 1990-1992 para o período 1993-1995. No estrato N/CO, os municípios que mostraram aumentos percentuais maiores do que $100 \%$ foram selecionados com probabi- 
lidade igual a um. No estrato S, foram escolhidos os municípios que mostraram aumentos percentuais mai ores do que $100 \%$ e com número acumulado de casos maior do que dez. No estrato RJ/SP, os municípios ou regiões compostos por bairros das duas capitais foram selecionados aleatoriamente. No segundo estágio, foram escolhidas as Comissões de Seleção de cada Região Militar com probabilidade proporcional ao tamanho em relação ao número de conscritos no ano de 1997.

Do ponto de vista da análise dos dados, é importante enfatizar dois aspectos relativos ao procedimento de amostragem:

1) os resultados aqui apresentados por estrato, apesar das denominações dadas a estes, não representam resultados das regiões brasileiras designadas pelos mesmos nomes. Convém lembrar ainda que somente os municípios com grandes aumentos relativos das taxas de incidência de AIDS foram selecionados para participar da pesquisa;

2) da mesma forma, os resultados apresentados como total da amostra não representam o total do Brasil.

O estudo contemplou um questionário autopreenchido pelos conscritos no momento de sua apresentação para o Exército e de coleta de sangue para teste de infecção pelo HIV. O questionário incluiu informações concernentes às características sócio-demográficas, práticas de comportamento sexual, problemas relacionados às infecções sexualmente transmissíveis (IST) e uso de drogas injetáveis. O questionário completo encontra-se disponível para consulta na home-page da CNDST/ AIDS (www.aids.gov. br), sob o título "Pesquisa Comportamental dos Conscritos do Exército do Brasil, 1998".

Dos conscritos selecionados coletaram-se alíquotas de sangue que foram submetidas a dois ensaios imunoenzimáticos (ELISA) com princípios metodológi cos e antígenos diferentes para detectar a infecção pelo HIV. Nessa etapa, as amostras indeterminadas, divergentes e reagentes foram testadas por imunoflorescência indireta - IFI. Aquel as que apresentaram resultados indeterminados ou negativos na reação de IFI, foram analisadas pelo teste de Western Blot, de acordo com a padronização disposta na Portaria 488/ 98 da Secretaria de Vigilância Sanitária do Ministério da Saúde, de 17 de junho de 1998. Todos os ensaios foram realizados no Laboratório do Instituto de Biologia do Exército Brasileiro, na cidade do Rio de Janeiro. Resultante de condições adversas, situação comum neste tipo de trabalho, somente 29.394 amostras puderam ser adequadamente processadas em termos laboratoriais.
Tendo em vista o objetivo do presente trabalho, a primeira etapa da análise estatística dos dados consistiu em elaborar estatísticas descritivas das principais características sócioeconômicas de acordo com os estratos. A análise das informações relativas à renda mensal familiar indicou que apenas $45 \%$ dos conscritos responderam a esta questão. Constatandose a impossibilidade de empregar esta variável como indicador de estratificação social, optouse por utilizar o grau de escolaridade (1o grau incompleto, 10 grau completo e 20 grau completo) como indicador do nível sócio-econômiCO (NSE).

A escolha deste indicador como aproximação para mensurar o NSE encontrou fundamentos na análise da renda média e mediana segundo o grau de escolaridade. Os dados dispostos na Tabela 1 demonstram a tendência crescente de renda à medida que o nível de instrução é mais elevado em todos os estratos considerados.

A seguir, foram investigadas todas as variáveis relativas ao comportamento sexual dos conscritos. Para tal, foram utilizados os seguintes indicadores:

1) Taxa de atividade sexual - definida como a proporção de conscritos que já tiveram relações sexuais.

2) Idade da primeira relação sexual.

Tabela 1

Renda média e mediana segundo o grau de escolaridade e o estrato. Brasil, 1998.

\begin{tabular}{lcrc}
\hline Estrato & Renda média (R\$) & \multicolumn{1}{c}{$n$} & Renda mediana (R\$) \\
\hline N/CO & & & \\
1o grau incompleto & 128,4 & 2213 & 70,0 \\
1o grau completo & 192,6 & 1515 & 100,0 \\
2o grau completo & 358,9 & 550 & 200,0 \\
Total & 180,8 & 4278 & 89,4 \\
& & & \\
RJ /SP & & & \\
1ㅇg grau incompleto & 178,1 & 1839 & 120,0 \\
1ㅇg grau completo & 300,3 & 1937 & 200,0 \\
2o grau completo & 539,8 & 696 & 300,0 \\
Total & 287,3 & 4472 & 166,7 \\
& & & \\
S & & & \\
1o grau incompleto & 170,7 & 1898 & 116,7 \\
1o grau completo & 274,1 & 1856 & 180,0 \\
2o grau completo & 469,2 & 734 & 283,3 \\
Total & 262,3 & 4488 & 160,0 \\
\hline
\end{tabular}


3) Número total de parceiros na vida, no último ano e nas últimas quatro semanas (anteriores à data da pesquisa).

4) Número de parceiros no último ano, conforme os seguintes tipos de parceria: parceiro(a) fixo(a); parceiro(a) casual - amigo(a), conhecido(a), paquera; parceiro(a) pago(a) - o conscrito pagou ao(à) parceiro(a) para ter relações sexuais; parceiro(a) pagante - o(a) parceiro(a) pagou ao conscrito para ter relações sexuais.

5) Percentual de conscritos que usaram preservativo na última relação sexual;

6) Percentual de conscritos que usaram preservativo em todas as relações sexuais no último ano de acordo com o tipo de parceria;

7) Percentual de relações sexuais nas últimas quatro semanas com o uso de preservativo;

Adicionalmente foram criados dois indicadores, visando expressar "comportamento sexual de risco":

8) Índice de comportamento sexual de risco no último ano (ICSR) - definido pela média ponderada do número de parceiros no ano anterior, tomando-se, como ponderações, as freqüências percentuais de não-uso de preservativo segundo o tipo de parceria. Matematicamente, pode-se expressar o ICSR por:

$\operatorname{ICSR}=\sum_{i=1}^{4}\left(1-w_{i}\right) \cdot P_{i}$

onde $\mathrm{i}$ indica o tipo de parceria;

$\mathrm{w}_{\mathrm{i}}=0,00$ - nunca usou preservativo com parceiros da categoria i;

$\mathrm{w}_{\mathrm{i}}=0,25$ - usou em menos da metade das vezes com parceiros da categoria i;

$\mathrm{w}_{\mathrm{i}}=0,75$ - usou em mais da metade das vezes com parceiros da categoria i;

$\mathrm{w}_{\mathrm{i}}=1,00$ - usou em todas as vezes com parceiros da categoria i;

$\mathrm{P}_{\mathrm{i}}=$ número de parceiros da categoria $\mathrm{i}$.

9) Í ndice de comportamento sexual de risco temporal (ICSRT) - definido pelo índice de comportamento sexual de risco no último ano multiplicado pelo tempo de exposição, calculado como a diferença entre a idade do conscrito e a idade da primeira relação sexual. Isto é:

ICSRT =ICSR $\times$ (idade - idade da 1a relação sexual).

Para a análise do uso de drogas injetáveis, utilizaram-se apenas as informações referentes à questão "você já usou drogas injetáveis?" para construir o indicador "Percentual de uso de drogas injetáveis".

No que se refere aos problemas relacionados às IST, os conscritos foram questionados quanto aos seguintes sinais: corrimento (pus) no canal da urina; feridas no pênis; pequenas bolhas no pênis; e verrugas no pênis, perguntando-se também com que idade ocorreram os problemas. Após a seleção prévia dos conscritos que declararam ter tido o problema após o início da atividade sexual, foram calculados os percentuais de ocorrência para cada um dos problemas. Além destes, foi construído o indicador "Percentual de ocorrência de pelo menos um problema relacionado às DST".

Todos os indicadores relativos às práticas sexuais, ao uso de drogas injetáveis e aos problemas relacionados às IST foram analisados segundo o grau de escolaridade por estrato, comparando-se as diferenças entre as proporções por categoria através de procedimentos de regressão logística, considerando-se como categorias-controle o estrato RJ/SP, para os estratos geográficos, e o segundo grau completo, para o grau de escolaridade. As diferenças nas médias foram testadas por procedimentos de análise de variância.

Para a ocorrência de um problema, pelo menos, relacionado às DST, foram estimados as odds ratios (OR), brutos e ajustados, por grau de escolaridade, estrato, índice de comportamento sexual de risco temporal, e uso de drogas injetáveis por meio de regressões logísticas uni e multivariadas.

Na segunda parte da análise, foram estimadas as proporções de infecção pelo HIV nos três estratos de estudo. Posteriormente, foram testados os efeitos (brutos e ajustados) das variáveis "grau de escolaridade”, "estrato", “índice de comportamento sexual de risco temporal" e "ocorrência de pelo menos um problema relacionado às IST", por meio de procedimentos de regressão logística, tendo como variável-resposta a infecção pelo HIV.

\section{Resultados}

Pelas estatísticas descritivas apresentadas na Tabela 2, nota-se que: mais da metade dos conscritos têm 18 anos de idade, sendo a amplitude de variação da idade de 17 a 21 anos; a grande maioria é de soltei ros e mora com a família; nos estratos RJ/SP e S, 40\% não finalizaram o 1o grau, enquanto este percentual é de $53 \%$ no estrato Norte/ Centro-Oeste. Ressalta-se que, neste último estrato, quase $70 \%$ das mães dos conscritos não completaram o 10 grau. Em relação à distribuição por renda, 55\% das perguntas não foram respondidas, o que impossibilitou análises posteriores segundo esta variável. Das respostas com informação de renda, 
mais de $45 \%$ têm renda familiar mensal até cinco salários mínimos em todos os estratos. Os conscritos que residem no estrato Norte/ Centro-Oeste possuíam a menor renda média familiar, sendo que $44 \%$ relataram renda familiar mensal inferior a três salários mínimos.

Alguns indicadores relacionados ao comportamento sexual dos conscritos segundo estrato e grau de escolaridade são apresentados na Tabela 3. Observa-se que o estrato N/CO é o que evidencia a maior taxa de atividade sexual, a idade mais precoce de início de relacionamento sexual e o maior número médio de parceiros na vida, no último ano e nas últimas quatro semanas, mostrando-se significativas as diferenças em relação ao estrato RJ/SP. Quanto ao grau de escolaridade, grandes variações são encontradas, sobretudo entre os conscritos com primeiro grau incompleto e aqueles que finalizaram o segundo grau. Em todos os três estratos, os conscritos com maior nível de instrução são os que têm a menor taxa de atividade sexual, iniciam vida sexual mais tardiamente e relatam menor número de parceiros sexuais.

A apreciação das estatísticas relativas ao número de parceiros no último ano segundo o tipo de parceria (Tabela 4) revela que o número médio de parceiro fixos nos últimos 12 meses é coerentemente próximo de um em todos os três estratos. O número médio de parceiros casuais varia de 1,2 (estrato RJ/SP) a 1,7 (N/CO). Da mesma forma, o menor número médio de parceiros pagos ou pagantes é encontrado no estrato RJ/SP e o maior no estrato N/CO. O Sul mostra-se sempre como estrato intermediário. No que se refere ao número médio de parceiros casuais, foram identificadas diferenças estatisticamente significativas na comparação dos estratos N/ CO e S com o estrato-controle (RJ/SP), mas não foram constatados efeitos significativos por nível de instrução. O grau de escolaridade demonstrou influenciar o percentual de parceiros fixos, que foi significativamente maior entre os conscritos de alta escolaridade.

Em todos os estratos, a proporção de conscritos que relataram ter usado preservativo na última relação sexual foi relativamente elevada. O menor percentual correspondeu ao estrato N/CO, $51 \%$, alcançando $67 \%$ nos outros dois estratos geográficos (Tabela 5). O percentual de relações sexuais com o uso do preservativo masculino nas últimas quatro semanas situou-se em patamar ligeiramente inferior, sobretudo nos estratos RJ/SP e S. Já o percentual de uso em todas as relações sexuais, no último ano, foi bem menor, com amplitude de variação de $30 \%$, no estrato $\mathrm{N} / \mathrm{CO}$, e $42 \%$, nos estratos RJ/SP e S. Na anál ise comparativa dos três in-
Tabela 2

Distribuição percentual dos conscritos segundo as categorias das variáveis sócio-demográficas e estrato. Brasil, 1998.

\begin{tabular}{|c|c|c|c|}
\hline \multirow[t]{2}{*}{ Variável } & \multicolumn{3}{|c|}{ Proporção (\%) por categoria } \\
\hline & $\mathrm{N} / \mathrm{CO}$ & $\mathrm{RJ} / \mathrm{SP}$ & $\mathrm{S}$ \\
\hline \multicolumn{4}{|l|}{ Idade (anos) } \\
\hline 17 & 15,6 & 24,5 & 30,1 \\
\hline 18 & 55,3 & 61,7 & 64,7 \\
\hline 19 & 18,3 & 7,8 & 3,7 \\
\hline 20 & 5,6 & 2,5 & 0,8 \\
\hline 21 & 5,1 & 3,5 & 0,7 \\
\hline \multicolumn{4}{|l|}{ Estado civil } \\
\hline solteiro & 92,6 & 95,5 & 95,2 \\
\hline casado ou amigado & 6,5 & 4,0 & 4,4 \\
\hline separado & 0,9 & 0,5 & 0,4 \\
\hline \multicolumn{4}{|l|}{ Escolaridade } \\
\hline 1o grau incompleto & 53,0 & 39,5 & 40,3 \\
\hline 10 grau completo & 34,9 & 44,5 & 42,1 \\
\hline 2o grau completo & 12,1 & 16,0 & 17,6 \\
\hline \multicolumn{4}{|l|}{ Escolaridade da mãe } \\
\hline Analfabeta & 12,7 & 12,1 & 9,0 \\
\hline 1o grau incompleto & 54,6 & 52,0 & 57,7 \\
\hline 1o grau completo & 14,4 & 13,2 & 12,7 \\
\hline 20 grau completo & 12,5 & 12,6 & 11,7 \\
\hline 3o grau completo & 5,8 & 10,1 & 8,8 \\
\hline \multicolumn{4}{|l|}{ Com quem mora } \\
\hline família & 90,6 & 92,7 & 91,8 \\
\hline sozinho & 2,7 & 2,0 & 2,5 \\
\hline parceira & 4,9 & 3,5 & 3,9 \\
\hline parceiro & 0,2 & 0,2 & 0,1 \\
\hline amigo & 1,7 & 1,5 & 1,7 \\
\hline \multicolumn{4}{|l|}{$\begin{array}{l}\text { Renda mensal familiar } \\
\text { (em salários mínimos)* }\end{array}$} \\
\hline$\leq 1 \mathrm{SM}$ & 10,2 & 2,7 & 3,6 \\
\hline $1 \& 3$ SM & 34,0 & 18,9 & 23,0 \\
\hline $3 \& 5$ SM & 24,2 & 25,1 & 25,4 \\
\hline $5 \& 10 \mathrm{SM}$ & 17,8 & 29,1 & 27,8 \\
\hline 10 \& $20 \mathrm{SM}$ & 7,7 & 15,1 & 12,8 \\
\hline$<20 \mathrm{SM}$ & 6,1 & 9,2 & 7,5 \\
\hline Tamanho da amostra & 9.923 & 10.408 & 9.987 \\
\hline
\end{tabular}

* excluindo-se os questionários com renda ignorada (55\%). 
Taxa de atividade sexual, idade média da primeira relação sexual e número médio de parceiros (na vida, no último ano e nas últimas quatro semanas) segundo o grau de escolaridade e estrato. Brasil, 1998.

\begin{tabular}{|c|c|c|c|c|c|}
\hline \multirow[t]{2}{*}{ Estrato } & \multirow{2}{*}{$\begin{array}{l}\text { Taxa de atividade } \\
\text { sexual }(\%)\end{array}$} & \multirow{2}{*}{$\begin{array}{l}\text { Idade média da } \\
\text { primeira relação }\end{array}$} & \multicolumn{3}{|c|}{ Número médio de parceiros } \\
\hline & & & na vida & no último ano & $\begin{array}{l}\text { nas últimas } \\
\text { quatro semanas }\end{array}$ \\
\hline \multicolumn{6}{|l|}{$\mathrm{N} / \mathrm{CO}$} \\
\hline 1 o grau incompleto & 89,9 & 14,50 & 8,68 & 3,86 & 1,66 \\
\hline lo grau completo & 86,6 & 14,58 & 8,46 & 3,75 & 1,47 \\
\hline 2 o grau completo & 85,6 & 14,78 & 8,46 & 3,18 & 1,34 \\
\hline Total & 88,2 & 14,56 & 8,58 & 3,73 & 1,55 \\
\hline \multicolumn{6}{|l|}{$\mathrm{RJ} / \mathrm{SP}$} \\
\hline 10 grau incompleto & 86,5 & 14,65 & 7,19 & 3,08 & 1,44 \\
\hline 1o grau completo & 80,2 & 14,88 & 5,61 & 2,57 & 1,25 \\
\hline 2o grau completo & 76,8 & 15,27 & 5,69 & 2,49 & 1,20 \\
\hline Total & 82,1 & 14,84 & 6,26 & 2,74 & 1,31 \\
\hline \multicolumn{6}{|l|}{ S } \\
\hline 1ㅇg grau incompleto & 84,9 & 14,51 & 7,73 & 3,36 & 1,47 \\
\hline 1o grau completo & 79,4 & 14,84 & 6,41 & 2,78 & 1,22 \\
\hline 2o grau completo & 76,9 & 15,25 & 5,98 & 2,88 & 1,20 \\
\hline Total & 81,2 & 14,77 & 6,88 & 3,02 & 1,32 \\
\hline \multicolumn{6}{|l|}{$\begin{array}{l}\text { Níveis de significância } \\
\text { descritivos dos efeitos }\end{array}$} \\
\hline \multicolumn{6}{|l|}{ Estrato } \\
\hline $\mathrm{N} / \mathrm{CO}$ & $<0,001$ & $<0,001$ & $<0,001$ & $<0,001$ & $<0,001$ \\
\hline $\mathrm{RJ} / \mathrm{SP}$ & - & - & - & - & - \\
\hline $\mathrm{S}$ & NS & 0,005 & $<0,001$ & 0,003 & NS \\
\hline \multicolumn{6}{|l|}{ Grau de escolaridade } \\
\hline 10 grau incompleto & $<0,001$ & $<0,001$ & $<0,001$ & $<0,001$ & $<0,001$ \\
\hline 10 grau completo & $<0,001$ & $<0,001$ & NS & NS & NS \\
\hline 2o grau completo & - & - & - & - & - \\
\hline
\end{tabular}

dicadores referentes à freqüência de uso de preservativo por nível de instrução, o grupo com menos anos de estudo mostrou diferenças significativas em relação aos demais.

Os dados dispostos na Tabela 6 mostram que, nos três estratos, a freqüência de uso de preservativo em todas as relações sexuais nos últimos 12 meses diminui à medida que o parceiro é fixo, aumentando quando o parceiro é casual. Cresce mais ainda quando o parceiro é pagante e atinge o máximo quando o parceiro é pago. De modo geral, a proporção de uso de preservativo cresce com o grau de escolaridade e o menor percentual de uso é sempre encontrado no estrato N/CO, independentemente do nível de instrução. Os resultados da análise es- tatística multivariada indicam efeitos significativos para ambos os níveis de escolaridade, para o tipo de parceria (fixo/ não fixo) e para o estrato N/CO em relação ao estrato-controle.

Os índices de comportamento sexual de risco, construídos a partir das informações concernentes ao número de parceiros e freqüência de uso de preservativo segundo o tipo de parceria, corroboram os resultados encontrados anteriormente para os outros indicadores ( Tabela 7). Diferenças significativas são evidenciadas tanto por estrato como por nível de escolaridade, destacando-se que o estrato $\mathrm{N} / \mathrm{CO}$ e o grupo com primeiro grau incompleto são as categorias que apresentam os piores índices de risco. 
Número médio de parceiros por tipo de parceria e percentual de parceiros fixos no último ano segundo o grau de escolaridade e o estrato. Brasil, 1998.

\begin{tabular}{|c|c|c|c|c|c|}
\hline \multirow[t]{2}{*}{ Estrato } & \multicolumn{4}{|c|}{ Número médio de parceiros } & \multirow{2}{*}{$\begin{array}{l}\text { Percentual }(\%) \text { de } \\
\text { parceiros fixos }\end{array}$} \\
\hline & fixos & casuais & pagos & pagantes & \\
\hline \multicolumn{6}{|l|}{$\mathrm{N} / \mathrm{CO}$} \\
\hline 10 grau incompleto & 0,90 & 1,79 & 0,48 & 0,10 & 23,3 \\
\hline 10 grau completo & 1,12 & 1,82 & 0,23 & 0,10 & 29,8 \\
\hline $2 \underline{0}$ grau completo & 1,21 & 1,71 & 0,19 & 0,07 & 38,0 \\
\hline Total & 1,02 & 1,79 & 0,35 & 0,09 & 27,4 \\
\hline \multicolumn{6}{|l|}{$\mathrm{RJ} / \mathrm{SP}$} \\
\hline 1o grau incompleto & 0,94 & 1,38 & 0,36 & 0,06 & 30,5 \\
\hline 1o grau completo & 1,07 & 1,29 & 0,25 & 0,06 & 41,4 \\
\hline 2o grau completo & 1,08 & 1,24 & 0,18 & 0,03 & 43,6 \\
\hline Total & 1,02 & 1,31 & 0,28 & 0,05 & 37,2 \\
\hline \multicolumn{6}{|l|}{ S } \\
\hline 10 grau incompleto & 0,98 & 1,64 & 0,39 & 0,11 & 29,2 \\
\hline 1 ㅇ grau completo & 1,16 & 1,56 & 0,21 & 0,07 & 41,8 \\
\hline 20 grau completo & 1,05 & 1,58 & 0,30 & 0,02 & 36,5 \\
\hline Total & 1,07 & 1,59 & 0,30 & 0,08 & 35,5 \\
\hline \multicolumn{6}{|l|}{$\begin{array}{l}\text { Níveis de significância } \\
\text { descritivos dos efeitos }\end{array}$} \\
\hline \multicolumn{6}{|l|}{ Estrato } \\
\hline $\mathrm{N} / \mathrm{CO}$ & NS & $<0,001$ & NS & 0,008 & $<0,001$ \\
\hline $\mathrm{RJ} / \mathrm{SP}$ & - & - & - & - & - \\
\hline $\mathrm{S}$ & 0,046 & $<0,001$ & NS & NS & 0,002 \\
\hline \multicolumn{6}{|l|}{ Grau de escolaridade } \\
\hline 1o grau incompleto & $<0,001$ & NS & $<0,001$ & 0,002 & $<0,001$ \\
\hline 10 grau completo & NS & NS & NS & 0,027 & 0,026 \\
\hline $2 \underline{o}$ grau completo & - & - & - & - & - \\
\hline
\end{tabular}

As informações contidas na Tabela 8 revelam que as proporções de conscritos que já fizeram uso de drogas injetáveis são semelhantes nos estratos N/CO e Sul (2,2\%), superiores à encontrada no estrato RJ/SP (1,4\%). Em todos os estratos, a proporção de conscritos que já usaram drogas injetáveis diminui quando cresce o grau de escolaridade. Constata-se que no estrato Sul são observadas as maiores proporções de uso para os dois níveis inferiores de instrução, 1o grau incompleto (3,3\%) e 1o grau completo (1,9\%).

Os problemas no pênis relatados pelos conscritos podem ser vistos como sinais de IST, em particular, o corrimento no canal urinário e as verrugas no pênis. Verifica-se que o estrato com predominância de problemas foi o N/CO, onde $8 \%$ relataram corrimento no canal urinário. O percentual de conscritos que relataram ter tido pelo menos um problema relacionado às IST foi relevante em todos os três estratos, superior a $10 \%$, atingindo valor maior do que $20 \%$ no N/CO. Os problemas mais citados foram bolhas e feridas no pênis, enquanto o menos freqüente foi a ocorrência de verrugas (Tabela 9 ).

Os resultados do procedimento de regressão logística, apresentados na Tabela 10, mostram associações significativas entre o grau de escolaridade e a ocorrência de, pelo menos, um problema relacionado às IST. Observa-se que, para a variável "corrimento no canal urinário", 
Percentual de conscritos que usaram preservativo na última relação sexual, em todas as vezes no último ano, percentual de relações sexuais com o uso de preservativo nas últimas quatro semanas segundo o grau de escolaridade e estrato. Brasil, 1998.

\begin{tabular}{|c|c|c|c|}
\hline Estrato & $\mathrm{Na}$ última relação (\%) & $\begin{array}{l}\text { Em todas as vezes } \\
\text { no último ano }(\%)\end{array}$ & $\begin{array}{l}\text { Percentual de relações } \\
\text { sexuais com preservativo } \\
\text { nas últimas quatro semanas }\end{array}$ \\
\hline \multicolumn{4}{|l|}{$\mathrm{N} / \mathrm{CO}$} \\
\hline 10 grau incompleto & 50,8 & 31,2 & 49,9 \\
\hline 1o grau completo & 50,5 & 29,9 & 53,6 \\
\hline 2o grau completo & 54,6 & 29,3 & 51,0 \\
\hline Total & 51,2 & 30,5 & 51,5 \\
\hline \multicolumn{4}{|l|}{$\mathrm{RJ} / \mathrm{SP}$} \\
\hline 1o grau incompleto & 63,5 & 37,5 & 54,8 \\
\hline 1o grau completo & 69,8 & 42,8 & 67,0 \\
\hline 2o grau completo & 72,6 & 48,4 & 64,9 \\
\hline Total & 67,7 & 42,1 & 62,0 \\
\hline \multicolumn{4}{|l|}{ S } \\
\hline lo grau incompleto & 62,6 & 37,8 & 50,0 \\
\hline 1o grau completo & 67,4 & 42,2 & 63,2 \\
\hline 2 o grau completo & 74,5 & 48,4 & 65,0 \\
\hline Total & 66,6 & 41,9 & 58,1 \\
\hline \multicolumn{4}{|l|}{$\begin{array}{l}\text { Níveis de significância } \\
\text { descritivos dos efeitos }\end{array}$} \\
\hline \multicolumn{4}{|l|}{ Estrato } \\
\hline $\mathrm{N} / \mathrm{CO}$ & $<0,001$ & $<0,001$ & $<0,001$ \\
\hline $\mathrm{RJ} / \mathrm{SP}$ & - & - & - \\
\hline $\mathrm{S}$ & NS & NS & $<0,001$ \\
\hline \multicolumn{4}{|l|}{ Grau de escolaridade } \\
\hline 1o grau incompleto & $<0,001$ & $<0,001$ & $<0,001$ \\
\hline 1o grau completo & $<0,001$ & $<0,001$ & NS \\
\hline 2 o grau completo & - & - & - \\
\hline
\end{tabular}

comparando-se os conscritos com primeiro grau incompleto àqueles que têm segundo grau completo, a razão ajustada dos produtos cruzados atinge 2,8. Chamam também a atenção as associações significativas e positivas encontradas para o índice de comportamento sexual de risco temporal, para todos os quatro problemas, que indicam que quanto maior o índice maior é a freqüência de ocorrência de um problema associado às IST.

As estimativas das proporções de infecção por HIV mostram diferenças relevantes entre os estratos (Tabela 11). O estrato RJ/ SP é o que apresenta a proporção mais baixa $(0,081 \%)$, significativamente menor que a encontrada no estrato N/CO (0,194\%). Resultado intermediário foi encontrado para o estrato S $(0,124 \%)$.

Os efeitos das variáveis "estrato", "grau de escolaridade", "índice de comportamento sexual de risco temporal (ICSRT)", e "ocorrência de pelo menos um problema relacionado às IST" na variável-resposta "presença de infecção pelo HIV", estimados por modelos de regressão logística múltipla, estão apresentados na Tabela 12. Os efeitos brutos correspondentes ao estrato N/CO ( $p=0,037)$, ao ICSRT $(p=0,004)$ e à ocorrência de pelo menos um problema relacionado às IST $(p \leq 0,001)$ foram estatistica- 
mente significativos. No entanto, o efeito ajustado correspondente ao estrato N/CO perde a significância estatística após o controle dos efeitos das outras variáveis consideradas no modelo.

\section{Discussão}

O plano de amostragem realizado nesta investigação, seguindo uma estratificação dos municípios brasileiros de acordo com o crescimento nas taxas de incidência de AIDS na década de 1990, permitiu evidenciar contrastes importantes que merecem particular consideração. Os achados indicam que o estrato N/CO - onde foi constatado grande aumento relativo das taxas de incidência de AIDS entre os períodos 1990-92 e 1993-95 (Szwarcwald et al., 1998) - é o que apresenta os indicadores mais baixos, sejam os relativos ao nível sócio-econômico, aos comportamentos sexuais de risco, ou aos problemas relacionados às IST. Esse estrato tem, em média, o mais baixo nível de instrução, o maior número de parceiros não fixos, as menores proporções de uso de preservativo (qualquer que seja a forma de mensuração do uso), o maior percentual de conscritos que fizeram uso de droga injetável eas maiores proporções de jovens que relataram problemas associados às infecções sexualmente transmissíveis.

Esses resultados adquirem expressão ainda mais importante quando as informações são anal isadas em conjunto com a presença de infecção por HIV. Foi também no estrato N/CO que se encontrou a maior proporção de infectados por HIV, associada, de modo significativo, à ocorrência de pelo menos um problema relacionado às IST e ao maior índice de comportamento sexual de risco.

Pesquisas realizadas com recrutas na Tailândia demonstraram resultados semelhantes quanto às diferenças regionais. Estudo de seguimento de recrutas mostrou contrastes regionais importantes na soro-incidência de infecção pelo HIV naquele país, com taxas 6 vezes maiores na Região Norte Superior $(3,2 \%)$ do que em Bangkok (0,5\%). As taxas de incidência foram significativamente menores nos adultos jovens casados no momento do alistamento e naqueles com mais de dez anos de instrução (Carr et al., 1994).

Na presente investigação, destaca-se igualmente o papel do grau de escolaridade como variável explicativa das diferenças referentes às práticas sexuais de risco dos conscritos. Sem exceção, em todos os estratos, os conscritos com primeiro grau incompleto têm início mais
Tabela 6

Proporção de conscritos que relataram ter usado camisinha em todas as relações sexuais no último ano segundo a escolaridade, o tipo de parceiro e o estrato. Brasil, 1998

\begin{tabular}{|c|c|c|c|c|}
\hline \multirow[t]{2}{*}{ Tipo de parceiro } & \multicolumn{3}{|c|}{ Proporção (\%) } & \multirow[t]{2}{*}{ Total } \\
\hline & $\mathrm{N} / \mathrm{CO}$ & $\mathrm{RJ} / \mathrm{SP}$ & S & \\
\hline \multicolumn{5}{|l|}{ Parceiro fixo } \\
\hline 10 grau incompleto & 33,2 & 40,1 & 41,5 & 38,1 \\
\hline 10 grau completo & 31,5 & 47,2 & 45,6 & 42,3 \\
\hline $2 \underline{o}$ grau completo & 31,5 & 51,5 & 50,2 & 45,6 \\
\hline Total & 32,2 & 46,0 & 45,3 & 41,6 \\
\hline \multicolumn{5}{|l|}{ Parceiro casual } \\
\hline 10 grau incompleto & 41,4 & 53,9 & 54,9 & 48,9 \\
\hline 1o grau completo & 44,0 & 62,4 & 59,3 & 55,6 \\
\hline $2 \underline{o}$ grau completo & 46,4 & 69,1 & 69,2 & 62,6 \\
\hline Total & 43,1 & 60,8 & 59,8 & 54,2 \\
\hline \multicolumn{5}{|l|}{ Parceiro pago } \\
\hline 10 grau incompleto & 53,7 & 63,9 & 60,1 & 58,3 \\
\hline 10 grau completo & 69,5 & 85,4 & 76,0 & 77,6 \\
\hline 2 o grau completo & 79,0 & 91,4 & 82,6 & 84,8 \\
\hline Total & 60,7 & 77,4 & 69,9 & 69,1 \\
\hline \multicolumn{5}{|l|}{ Parceiro pagante } \\
\hline 1o grau incompleto & 50,4 & 61,5 & 60,5 & 55,8 \\
\hline 10 grau completo & 46,5 & 75,4 & 63,5 & 58,7 \\
\hline 2 o grau completo & 50,0 & 71,4 & 70,0 & 60,0 \\
\hline Total & 48,8 & 69,1 & 61,8 & 57,3 \\
\hline \multicolumn{5}{|l|}{$\begin{array}{l}\text { Níveis de significância } \\
\text { descritivos dos efeitos }\end{array}$} \\
\hline \multicolumn{5}{|l|}{ Estrato } \\
\hline $\mathrm{N} / \mathrm{CO}$ & & & & $<0,001$ \\
\hline $\mathrm{RJ} / \mathrm{SP}$ & & & & - \\
\hline $\mathrm{S}$ & & & & NS \\
\hline \multicolumn{5}{|l|}{ Grau de escolaridade } \\
\hline 1o grau incompleto & & & & $<0,001$ \\
\hline 1o grau completo & & & & $<0,001$ \\
\hline $2 \underline{o}$ grau completo & & & & - \\
\hline \multicolumn{5}{|l|}{ Tipo de Parceiro } \\
\hline Fixo & & & & 0,004 \\
\hline Não fixo & & & & - \\
\hline
\end{tabular}


Tabela 7

Índice médio de comportamento sexual de risco por grau de escolaridade e estrato. Brasil, 1998.

\begin{tabular}{|c|c|c|}
\hline Estrato & Índice médio & Índice médio temporal \\
\hline \multicolumn{3}{|l|}{$\mathrm{N} / \mathrm{CO}$} \\
\hline 1o grau incompleto & 1,46 & 5,96 \\
\hline 1o grau completo & 1,32 & 5,62 \\
\hline 2 o grau completo & 1,28 & 5,59 \\
\hline Total & 1,38 & 5,79 \\
\hline \multicolumn{3}{|l|}{$\mathrm{RJ} / \mathrm{SP}$} \\
\hline 1o grau incompleto & 1,00 & 4,14 \\
\hline 1ㅇg grau completo & 0,87 & 2,87 \\
\hline $2 \underline{0}$ grau completo & 0,76 & 2,53 \\
\hline Total & 0,90 & 3,28 \\
\hline \multicolumn{3}{|l|}{$\mathrm{S}$} \\
\hline 10 grau incompleto & 1,15 & 4,26 \\
\hline 10 grau completo & 0,94 & 3,09 \\
\hline 2 o grau completo & 0,71 & 2,06 \\
\hline Total & 0,98 & 3,35 \\
\hline \multicolumn{3}{|l|}{$\begin{array}{l}\text { Níveis descritivos de } \\
\text { significância dos efeitos }\end{array}$} \\
\hline \multicolumn{3}{|l|}{ Estrato } \\
\hline $\mathrm{N} / \mathrm{CO}$ & $<0,001$ & $<0,001$ \\
\hline $\mathrm{RJ} / \mathrm{SP}$ & - & - \\
\hline $\mathrm{S}$ & NS & NS \\
\hline \multicolumn{3}{|l|}{ Grau de escolaridade } \\
\hline 10 grau incompleto & $<0,001$ & $<0,001$ \\
\hline 1ㅇg grau completo & $<0,001$ & 0,022 \\
\hline 2 o grau completo & - & - \\
\hline
\end{tabular}

precoce de relacionamento sexual, apresentam a taxa mais elevada de atividade sexual, o maior número de parceiros casuais, as menores freqüências de uso de preservativo, o que implica os piores índices de comportamento sexual de risco. Por sua vez, estes últimos mostraram-se importantes preditores dos problemas relacionados às infecções sexualmente transmissíveis, incluindo a infecção por HIV.

Esses resultados corroboram aqueles de estudos feitos em outros países, os quais expressam que os jovens adolescentes de baixa instrução e nível sócio-econômico precário são os mais suscetíveis às IST (O'Leary et al., 1998). As explicações recaem no comportamento sexual de maior risco (Fishbein et al., 1993), nas dificuldades de controlar o impulso sexual (LoConte et al., 1997) e no menor conhecimento a respeito das doenças e das medidas de prevenção (Ford \& Norris, 1993)

No Brasil, pesquisas realizadas com adolescentes mostram relativo grau de conhecimento acerca das formas de transmissão do HIV/AIDS, que é tanto melhor quanto maior o grau de escolaridade. A Pesquisa Nacional sobre Demografia e Saúde (PNDS) revelou que apenas 9,5\% dos adolescentes brasileiros de 15 a 19 anos ignoram qualquer maneira de evitar a transmissão do HIV/ AIDS. Porém, analisando os resultados por anos de educação, este percentual variou de $36 \%$, entre os sem instrução, a 0,1\%, entre aqueles com 12 anos ou mais de escolaridade (BEMFAM, 1997b).

A implementação de programas para prevenir comportamentos sexuais de risco é atualmente considerada prioridade na área de saúde do adolescente (Genuis \& Genuis, 1995; DiClemente, 1998). O percentual, ainda pequeno, de uso de preservativos em todas as relações sexuais por parte dos jovens brasileiros - que não ultrapassa o patamar de $40 \%$, e não parece ter aumentado significativamente nos últimos anos (CNDST/ AIDS, 1998a; 1998b) - indica que esforços, em nível nacional, devem ser dirigidos à compreensão dos adolescentes quanto aos possíveis efeitos de comportamentos sexuais de risco, decorrentes muitas vezes de impulsos sem reflexão ou de influências de companheiros da escola ou da comunidade de residência (Kusseling et al., 1996). Ademais, os resultados da presente investigação redundam na necessidade de desenvolvimento de estratégias de intervenção dirigidas especialmente aos jovens em condições desvantajosas de vida, de baixo nível de instrução, que constituem o segmento populacional identificado como o de maior vulnerabilidade à disseminação da infecção pelo HIV e o mais difícil de ser atingido pelas medidas preventivas (Conway et al., 1993).

Programas de intervenção realizados em outros países com jovens pertencentes às minorias sociais indicaram que a divulgação do conhecimento é importante, mas não suficiente para causar impacto no comportamento de risco sexual (Walter \& Vaughn, 1993). Aspectos relacionados à percepção de vulnerabilidade e ao conceito de auto-eficácia, entendida como a competência individual em adotar práticas de comportamento especificamente para não contrair a infecção pelo HIV, vêm se mostrando fundamentais nas abordagens de motivação do uso de camisinha e da implementação das ações preventivas (Reitman et al., 1996; Ku et al., 1998). 
A mensagem utilizada nos programas de prevenção de comportamento de risco entre jovens é elemento que igualmente se destaca como fundamental. Pesquisadores têm demonstrado que ela deve ser modificada de acordo com as distintas culturas (Stanton et al., 1999), respeitando as diferenças de comportamento sexual, as crenças e os mitos peculiares aos distintos segmentos populacionais (Ford \& Norris, 1993). No caso particular dos adolescentes desfavorecidos, a linguagem precisa ser tanto sensível aos que têm educação limitada como também adequada àqueles que vivem em situação de marginalidade social (Organista et al., 1997).

No que se refere aos problemas relacionados às IST, foi notório o percentual mais elevado evidenciado no estrato N/CO - em especial, entre os conscritos com primeiro grau incompleto, grupo populacional que também apresentou a maior prevalência de infecção pelo HIV. Estes achados são similares aos encontrados com conscritos militares da Tailândia, que também apresentaram claras associações entre as IST e a incidência de infecção pelo HIV (Nelson et al., 1997; Nopkesorn et al., 1998).

Vários estudos apontam para a ocorrência de outras IST como fator de risco adicional para a infecção pelo HIV (Boily \& Anderson, 1996). Uma das razões levantadas é que a presença de uma IST em um dos parceiros potencializa o risco da transmissão do HIV (O'Leary et al., 1998). Por outro lado, não se pode deixar de considerar que os fatores de risco são os mesmos e que estes assumem ainda maior relevância em virtude dos efeitos da interação sexual sel etiva das redes sociais (Anderson \& Gupta, 1990; Morris, 1997).

A mesma racionalização pode ser feita no tocante a outros comportamentos de risco, como o uso de drogas (Friedman et al., 1997). Embora a transmissão do HIV ocorra primariamente entre os usuários de drogas injetáveis e seus parceiros sexuais, o uso de drogas por diferentes meios, como o crack e a cocaína "cheirada", é reconhecidamente vinculado às práticas sexuais desprotegidas (Fullilove \& Fullilove, 1989; Edlin et al., 1994). Como sugerido por alguns autores (Elwood et al., 1997; Perlman et al., 1999), o consumo de drogas ilícitas se associa ao risco aumentado de infecção pelo HIV, em particular, pelo comportamento sexual de risco em troca de drogas.

É importante enfatizar que, no caso particular deste estudo, as maiores proporções de uso de drogas injetáveis, para qualquer um dos três estratos, ocorreram nos grupos com menor escolaridade, sugerindo a exposição simultânea a múltiplos fatores: comportamento se-
Tabela 8

Proporção de conscritos que fizeram uso de drogas injetáveis segundo o estrato e a escolaridade, Brasil, 1998.

\begin{tabular}{|c|c|c|c|}
\hline \multirow[t]{2}{*}{ Escolaridade } & \multicolumn{3}{|c|}{ Estrato } \\
\hline & $\mathrm{N} / \mathrm{CO}$ & $\mathrm{RJ} / \mathrm{SP}$ & $\mathrm{S}$ \\
\hline 10 grau incompleto & $3,2 \%$ & $2,5 \%$ & $3,3 \%$ \\
\hline 1 o grau completo & $1,5 \%$ & $0,8 \%$ & $1,9 \%$ \\
\hline 2o grau completo & $0,8 \%$ & $0,3 \%$ & $0,8 \%$ \\
\hline Total & $2,3 \%$ & $1,4 \%$ & $2,2 \%$ \\
\hline \multicolumn{4}{|l|}{$\begin{array}{l}\text { Níveis de significância } \\
\text { descritivos dos efeitos }\end{array}$} \\
\hline \multicolumn{4}{|l|}{ Estrato } \\
\hline $\mathrm{N} / \mathrm{CO}$ & & & 0,001 \\
\hline $\mathrm{RJ} / \mathrm{SP}$ & & & - \\
\hline $\mathrm{S}$ & & & $<0,001$ \\
\hline \multicolumn{4}{|l|}{ Grau de escolaridade } \\
\hline 10 grau incompleto & & & $<0,001$ \\
\hline 10 grau completo & & & $<0,001$ \\
\hline $2 \underline{o}$ grau completo & & & - \\
\hline
\end{tabular}

xual de risco, outras IST e drogas, tal como descrito anteriormente por Des Jarlais et al. (1991).

Recentemente, vários autores - como Singer (1994) e Massey (1996) - têm apontado a sinergia dos efeitos adversos da concentração residencial de pobreza - em especial, nas áreas urbanas - como força primária na direção de exacerbar a disseminação das IST. O problema toma dimensão mais grave à medida que a disseminação facilmente ultrapassa as fronteiras das comunidades, já que muitos jovens sexualmente ativos de outros grupos sociais podem perfeitamente escolher parceiros sexuais dessas redes sócio-geográficas (Wallace \& Wallace, 1995; Friedman et al., 1997).

No que tange às limitações do presente estudo, deve-se ressaltar o tipo de amostragem utilizada. Embora, a estratificação tenha produzido resultados condizentes aos aumentos nas taxas de incidência de AIDS em anos recentes, os municípios selecionados não são representativos das regiões, assim como os três estratos não correspondem ao total do Brasil, e portanto, os resultados não podem ser consolidados para o País como um todo.

Ainda em relação à amostra, convém notar que o universo pesquisado muito grande - cerca de 10.000 em cada estrato - foi calculado com o objetivo de estimar diferenças nas soro- 
Percentual (\%) de conscritos que relataram ter tido problemas relacionados às infecções sexualmente transmissíveis por tipo de problema, segundo o grau de escolaridade e o estrato. Brasil, 1998.

\begin{tabular}{|c|c|c|c|c|c|}
\hline \multirow[t]{2}{*}{ Estrato } & \multicolumn{4}{|c|}{ Problemas relacionados às IST $(\%)$} & \multirow{2}{*}{$\begin{array}{l}\text { Pelo menos } \\
\text { um problema }\end{array}$} \\
\hline & corrimento & bolhas & feridas & verrugas & \\
\hline \multicolumn{6}{|l|}{$\mathrm{N} / \mathrm{CO}$} \\
\hline 10 grau incompleto & 9,5 & 9,0 & 8,4 & 3,5 & 22,3 \\
\hline 1o grau completo & 6,7 & 8,1 & 8,4 & 3,2 & 20,5 \\
\hline 2o grau completo & 4,5 & 9,2 & 6,9 & 2,9 & 18,5 \\
\hline Total & 7,9 & 8,7 & 8,2 & 3,4 & 21,2 \\
\hline \multicolumn{6}{|l|}{$\mathrm{RJ} / \mathrm{SP}$} \\
\hline 10 grau incompleto & 4,5 & 6,0 & 4,5 & 2,5 & 13,2 \\
\hline 1o grau completo & 1,7 & 4,0 & 4,0 & 1,6 & 9,3 \\
\hline 2o grau completo & 1,2 & 3,6 & 2,9 & 1,8 & 7,7 \\
\hline Total & 2,8 & 4,8 & 4,0 & 2,0 & 10,7 \\
\hline \multicolumn{6}{|l|}{ S } \\
\hline 1ㅇ grau incompleto & 4,8 & 5,4 & 5,3 & 2,2 & 13,7 \\
\hline 1o grau completo & 2,2 & 3,3 & 4,6 & 1,7 & 9,8 \\
\hline 2 o grau completo & 1,2 & 4,1 & 3,5 & 1,2 & 8,4 \\
\hline Total & 3,1 & 4,3 & 4,7 & 1,8 & 11,2 \\
\hline \multicolumn{6}{|l|}{$\begin{array}{l}\text { Níveis de significância } \\
\text { descritivos dos efeitos }\end{array}$} \\
\hline \multicolumn{6}{|l|}{ Estrato } \\
\hline $\mathrm{N} / \mathrm{CO}$ & $<0,001$ & $<0,001$ & $<0,001$ & $<0,001$ & $<0,001$ \\
\hline $\mathrm{RJ} / \mathrm{SP}$ & - & - & - & - & - \\
\hline $\mathrm{S}$ & NS & NS & 0,030 & NS & NS \\
\hline \multicolumn{6}{|l|}{ Grau de escolaridade } \\
\hline 1ㅇg grau incompleto & $<0,001$ & 0,012 & $<0,001$ & 0,015 & $<0,001$ \\
\hline 1o grau completo & 0,001 & NS & 0,006 & NS & 0,009 \\
\hline 20 grau completo & - & - & - & - & - \\
\hline
\end{tabular}

prevalências da infecção pelo HIV, que configuram proporções bem pequenas e requerem expressivos tamanhos de amostra para a estimação com certa precisão. Sendo assim, as várias inferências não relativas às proporções de infectados pelo HIV - em particular, as que conduziram à rejeição da hipótese nula - devem ser examinadas à luz desta limitação. Por outro lado, tal tamanho de amostra acarretou a estimação bastante precisa das médias e proporções, as quais devem ser, portanto, bem próximas dos valores populacionais.

Outro fator limitador da pesquisa foi decorrente da falta de preenchimento da variável "renda familiar" por mais da metade dos conscritos. Tendo em vista que o questionário não incluiu perguntas adicionais que pudessem medir indiretamente o padrão de vida dos jovens para fins do recorte sócio-econômico, o estudo teve que se restringir a empregar o grau de escolaridade, indicador reconhecidamente menos sensível aos efeitos adversos dos grupos de adolescentes socialmente marginalizados, principalmente os envolvidos em trabalho comercial de sexo, que vendem sexo em troca de drogas, favores ou dinheiro, e, muitas vezes, são forçados a consentir em relações desprotegidas (Peersman \& Levy, 1998).

É preciso tomar em conta também que não foram incluídos na investigação os conscritos analfabetos, em razão da impossibilidade de preencherem os questionários. Em conseqüên- 
Odds ratios (OR) ajustadas de "ter pelo menos um problema relacionado às infecções sexualmente transmissíveis". Brasil, 1998.

\begin{tabular}{|c|c|c|c|c|c|c|c|c|c|c|}
\hline \multirow[t]{2}{*}{ Variáveis } & \multicolumn{2}{|c|}{ Corrimento } & \multicolumn{2}{|c|}{ Feridas } & \multicolumn{2}{|c|}{ Bolhas } & \multicolumn{2}{|c|}{ Verrugas } & \multicolumn{2}{|c|}{$\begin{array}{l}\text { Pelo menos } \\
\text { um problema }\end{array}$} \\
\hline & OR & $p$ & OR & $\mathrm{p}$ & OR & $\mathrm{p}$ & OR & $\mathrm{p}$ & OR & $\mathrm{p}$ \\
\hline \multicolumn{11}{|l|}{ Grau de escolaridade } \\
\hline 1o grau incompleto & 2,8 & $<0,001$ & 1,2 & NS & 1,25 & NS & 1,23 & NS & 1,47 & $<0,001$ \\
\hline 1o grau completo & 1,62 & 0,002 & 0,91 & NS & 1,31 & 0,016 & 1,11 & NS & 1,21 & $<0,001$ \\
\hline 2o grau completo & 1,00 & - & 1,00 & - & 1,00 & - & - & - & 1,00 & - \\
\hline \multicolumn{11}{|l|}{ Estrato } \\
\hline $\mathrm{N} / \mathrm{CO}$ & 2,91 & $<0,001$ & 1,71 & NS & 1,90 & $<0,001$ & 1,89 & $<0,001$ & 2,04 & $<0,001$ \\
\hline $\mathrm{RJ} / \mathrm{SP}$ & 1,00 & - & 1,00 & - & 1,00 & - & 1,00 & - & 1,00 & - \\
\hline $\mathrm{S}$ & 1,17 & NS & 0,87 & NS & 1,09 & NS & 1,04 & NS & 1,01 & NS \\
\hline Índice de risco temporal & 1,02 & $<0,001$ & 1,02 & $<0,001$ & 1,01 & $<0,001$ & 1,02 & $<0,001$ & 1,03 & $<0,001$ \\
\hline UDI & 2,40 & $<0,001$ & 2,61 & $<0,001$ & 2,13 & $<0,001$ & 2,83 & $<0,001$ & 2,56 & $<0,001$ \\
\hline
\end{tabular}

$\mathrm{p}=$ nível de significância descritivo.

Tabela 11

Proporção (\%) de conscritos infectados pelo HIV segundo o estrato. Brasil, 1998.

\begin{tabular}{lcccc}
\hline Estrato & No de positivos & Tamanho da amostra & Proporção (\%) & $\begin{array}{c}\text { Intervalo de } 95 \% \\
\text { de confiança }\end{array}$ \\
\hline N/CO & 19 & 9.788 & 0,194 & $0,107-0,281$ \\
RJ /SP & 8 & 9.899 & 0,081 & $0,025-0,137$ \\
S & 12 & 9.707 & 0,124 & $0,054-0,194$ \\
\hline
\end{tabular}

cia, é provável que não tenham feito parte da presente análise aqueles que vivem nas piores situações de privação social. Vale notar, entretanto, que a ocorrência de sexo comercial mostrou-se associada à categoria de mais baixa instrução, embora a categorização do grau de escolaridade utilizada não seja suficientemente sensível para captar distintos níveis de pobreza.

Diferentemente das análises com casos notificados de AIDS que delineiam o perfil da infecção pelo HIV de 8 a 10 anos atrás (Deuffic \& Costagliola, 1999), as pesquisas com conscritos descrevem a situação atual em jovens do sexo masculino. Os resultados aqui depreendidos falam assim, de forma concreta, a favor da hipótese de pauperização da AIDS no Brasil, no sentido de que os jovens de mais baixo nível sócioeconômico passaram a ser os mais suscetíveis à infecção pelo HIV.
Dado que os jovens das classes sociais mais pobres são os que apresentam também a maior vulnerabilidade às IST e outros fatores de risco, aliado ao fato de que indivíduos de um segmento social tendem a interagir sexual mente com indivíduos daquele mesmo grupo, podese prever que os contrastes aqui encontrados determinarão dinâmicas distintas da epidemia de AIDS, em futuro próximo, expressas por processos de disseminação mais intensos na população com as piores condições sócio-econômicas.

Sob esta perspectiva, não se pode deixar de considerar as influências do ambiente social particularmente relevantes na determinação do comportamento de risco entre os adolescentes (Romer et al., 1994) - e, portanto, capazes de afetar os processos de disseminação das IST. Evidências neste sentido foram confirma- 
Tabela 12

Odds ratios (brutas e ajustadas) para a presença de infecção pelo HIV. Brasil, 1998.

\begin{tabular}{|c|c|c|c|c|c|c|}
\hline Variável & OR Bruta & IC $95 \%$ & $\begin{array}{l}\text { Nível de significância } \\
\text { descritivo }\end{array}$ & OR ajustada & IC $95 \%$ & Significância \\
\hline \multicolumn{7}{|l|}{ Estrato } \\
\hline $\mathrm{N} / \mathrm{CO}$ & 2,405 & $1,052-5,496$ & 0,037 & 1,477 & $0,479-4,551$ & NS \\
\hline $\mathrm{RJ} / \mathrm{SP}$ & 1,000 & - & - & 1,000 & - & - \\
\hline $\mathrm{S}$ & 1,530 & $0,625-3,745$ & NS & 0,958 & $0,277-3,312$ & NS \\
\hline \multicolumn{7}{|l|}{ Grau de escolaridade } \\
\hline lo grau incompleto & 1,635 & $0,556-4,802$ & NS & 0,746 & $0,184-3,015$ & NS \\
\hline 1o grau completo & 1,314 & $0,433-3,989$ & NS & 1,253 & $0,343-4,570$ & NS \\
\hline 2 o grau completo & 1,000 & - & - & 1,000 & - & - \\
\hline Índice de risco temporal & $1,017^{*}$ & $1,005-1,028$ & 0,004 & 1,001 & $1,001-1,026$ & 0,036 \\
\hline \multicolumn{7}{|l|}{$\begin{array}{l}\text { Pelo menos um problema } \\
\text { relacionado às DST }\end{array}$} \\
\hline $\operatorname{Sim}$ & 3,127 & $1,591-6,145$ & $<0,001$ & 2,784 & $1,042-7,444$ & 0,041 \\
\hline Não & 1,000 & - & - & - & - & - \\
\hline
\end{tabular}

* Para esta variável, a estimativa refere-se à exponencial do coeficiente correspondente na regressão logística.

das na presente investigação, já que os conscritos demonstraram comportamentos de risco diferenciado por região de residência, mesmo controlando-se pelo efeito da variável "nível de escolaridade".

Em termos conclusivos, os resultados desta pesquisa permitem identificar a necessidade da implementação de programas de prevenção, especificamente direcionados aos adolescentes e jovens adultos brasileiros. As medidas devem ser dirigidas não só a prevenir a infecção pelo HIV, mas compreender intervenções de redução das IST em geral (Mehret et al., 1996) e de outros fatores associados (Perlman et al., 1999), integrando a divulgação do conhecimento a respeito das IST e a abordagem de aspectos relacionados à percepção de vulnerabilidade (Poppen \& Reisen, 1997), à respon- sabilidade social (Waterson, 1997) e ao conceito de auto-eficácia (Buunk et al., 1998), de modo a motivar os jovens a mudar atitudes, a controlar impulsos e a restringir comportamentos de risco.

De forma a otimizar a abrangência no âmbito nacional, os programas devem envolver estratégias locais, em conformidade com as peculiaridades das regiões e comunidades, com enfoque particular em determinados segmentos populacionais, respeitando as mensagens de comunicação e as ações apropriadas a cada grupo social. Visando estabelecer o controle das distintas dinâmicas de disseminação da epidemia de AIDS no Brasil, um dos maiores desafios a ser enfrentado é assegurar o impacto generalizado das medidas, em especial, entre os mais suscetíveis.

\section{Referências}

ANDERSON, J. E.; KANN, L.; HOLTZM AN, D.; ARDAY, S.; TRUMAN, B. \& KOLBE, L., 1990. HIV/AIDS knowledge and sexual behavior among high school students. Family Planning Perspectives, 22:252255.

ANDERSON, R. M. \& GUPTA, S., 1990. The significance of sexual partner contact networks for the transmission dynamics of HIV. Journal of Acquired Immune Deficiency Syndromes, 3:417-429. 
BEMFAM (Sociedade Civil Bem-Estar Familiar no Brasil), 1997a. Brasil: Pesquisa Nacional sobre Demografia eSaúde 1996. Rio de Janeiro: BEMFAM.

BEMFAM (Sociedade Civil Bem-Estar Familiar no Brasil), 1997b. Brasil: Pesquisa Nacional sobre Demografia e Saúde: Uma Análise do Nível de Conhecimento eComportamentos deVulnerabilização. Rio de Janeiro: BEMFAM.

BOILY, M.-C. \& ANDERSON, R. M., 1996. Human Immunodeficiency Virus Transmission and the role of other sexually transmitted diseases. Sexually Transmitted Diseases, 23:312-332.

BUUNK, B. P.; BAKKER, A. B.; SIERO, F. W.; VAN DEN EIJNDEN, R. J. \& YZER, M. C., 1998. Predictors of AIDS-preventive behavioral intentions among adult heterosexuals at risk for HIV-infection: Extending current models and measures. AIDS Education and Prevention, 10:149-172.

CARR, J. K.; SIRISOPANA, N.; TORUGSA, K.; JUGSUDEE, A.; SUPAPONGSE, T.; CHUENCHITRA, C.; NITAYAPHAN, S.; SINGHARAJ, P. \& MCNEIL, J. G., 1994. Incidence of HIV-1 infection among young men in Thailand. Journal of Acquired Immune Deficiency Syndromes, 7:1270-1275.

CNDST/ AIDS (Coordenação Nacional de DST e AIDS), 1998a. Conhecimento sobre os Meios de Transmissão da AIDS: Uma Avaliação com Conscritos do Exército. Brasil, 1996. Brasília: Coordenação Nacional de DST e AIDS, Ministério da Saúde.

CNDST/AIDS (Coordenação Nacional de DST e AIDS), 1998b. Avaliação do Grau de Conhecimento eUso dePreservativo em Conscritos do Exército Brasileiro. Ano de Apresentação 1997. Brasília: Coordenação Nacional de DST e AIDS, Ministério da Saúde.

CONWAY, G. A.; EPSTEIN, M. R.; HAYMAN, C. R.; MILLER, C. A.; WENDELL, D. A.; GWINN, M.; KARON, J. M. \& PETERSEN, L. R., 1993. Trends in HIV prevalence among disadvantaged youth. JAMA, 269:2887-2889.

DES JARLAIS, D. C.; ABDUL-QUADER, A.; MINKOFF, H.; HOEGSBERG, B.; LANDESM AN, S. \& TROSS, S., 1991. Crack use and multiple AIDS risk behaviors. Journal of Acquired Immune Deficiency Syndromes, 4:446-447.

DEUFFIC, S. \& COSTAGLIOLA, D., 1999. Is the AIDS incubation time changing? A back-calculation approach. Statistics in Medicine, 18:1.031-1.047.

DICLEMENTE, R. J., 1998. Editorial: Preventing sexually transmitted infections among adolescents. A clash of ideology and science. JAM A, 279:15741575.

DICLEMENTE, R. J.; PORTON, L. E. \& HANSEN, W. B., 1996. New Directions for Adolescent Risk Prevention Research and Health Promotion Research and Interventions. Handbook of Adolescent Health Risk Behavior. Issues in Clinical Child Psychology. New York: Plenum Press.

EDLIN, B. R.; IRWIN, K. L.; FARUQUE, S.; MCCOY, C. B.;WORD, C.; SERRANO, Y.; INCIARDI, J. A.; BOWSER, B. P.; SCHILLING, R. F. \& HOLMBERG, S. D., 1994. Intersecting epidemics - Crack cocaine use and HIV infection among inner-city young adults. New England Journal of Medicine, 24:1422-1427.

EDLIN, B. R.; IRWIN, K. L.; FARUQUE, S.; MCCOY, C. B.;WORD, C.; SERRANO, Y.; INCIARDI, J. A.;
BOWSER, B. P.; SCHILLING, R. F. \& HOLMBERG, S. D., 1994. Intersecting epidemics - Crack cocaine use and HIV infection among inner-city young adults. New England Journal of Medicine, 24:1422-1427.

ELWOOD, W. N.; WILLIAMS, M. L.; BELL, D. C. \& RICHARD, A. J., 1997. Powerlessness and HIV prevention among people who trade sex for drugs ("strawberries"). AIDS Care, 9:273-284.

FISHBEIN, M.; DOUGLAS, J. M.; RHODES, F., HANANEL, L. D. \& NAPOLITANO, E., 1993. Distribution of STD clinic patients along a stages of behavior-change continuum - Selected sites, 1993. MMWR, 42:880-883.

FORD, K. \& NORRIS, A. E., 1993. Knowledge of AIDS transmission, risk behavior, and perceptions of risk among urban, low-income, African-American and Hispanic youth. American Journal of Preventive Medicine, 9:297-306.

FORD, K.; RUBINSTEIN, S. \& NORRIS, A. E., 1994. Sexual behavior and condom use among urban, low-income, African-American and Hispanic youth. AIDS Education and Prevention, 6:219229.

FRIEDMAN, S. R.; CURTIS, R.; JOSE, B.; NEAIGUS, A.; ZENILMAN, J.; CULPEPPER-MORGAN, J.; BORG, L.; KREEK, J.; PAONE, D. \& DES JARLAIS, D. C., 1997. Sex, drugs and infections among youth. Sexually Transmitted Diseases, 24:322-326.

FULLILOVE, M. T. \& FULLILOVE, R. E., 1989. Intersecting epidemics. Black teen crack and sexually transmitted diseases. Journal of the American Medical Women's Association, 44:146-153.

GENUIS, S. J. \& GENUIS, S. K., 1995. Adolescent sexual involvement: Time for primary prevention. Lancet, 345:240-241.

KANN, L.; KINCHEN, S. A.; WILLIAMS, B. I.; ROSS, J. G.; LOWRY, R.; HILL, C. V.; GRUNBAUM, J. A.; BLUMSON, P. S.; COLLINS, J. L. \& KOLBE, L. J., 1998. Youth risk behavior surveillance- United States, 1997. Journal of School Health, 68:355-369.

KU, L.; SONENSTEIN, F. L.; LINDBERG, L. D.; BRADNER, C. H.; BOGGESS, S. \& PLECK, J. H., 1998. Understanding changes in sexual activity among young metropolitan men: 1979-1995. Family Planning Perspectives, 30:256-262.

KUSSELING, F. S.; SHAPIRO, M. F.; GREENBERG, J. M. $\&$ WENGER, N. S., 1996. Understanding why heterosexual adults do not practice safer sex: A comparison of two samples. AIDS Education and Prevention, 8:247-257.

LOCONTE, J. S.; O'LEARY, A. \& LABOUVIE, E., 1997. Psychosocial correlates of HIV-related sexual behavior in an inner-city STD clinic. Psychology and Health, 12:589-601.

MASSEY, D. S., 1996. The age of extremes: Concentrated affluence and poverty in the twenty-first century. Demography, 33:395-412.

MEHRET, M.; MERTENS, T. E.; CARAËL, M.; NEGASSA, H.; FELEKE, W.; YITBAREK, N.; BURTON, T., 1996. Baseline for the evaluation of an AIDS programme using prevention indicators: A case study in Ethiopia. Bulletin of theWorld Health Organization, 74:509-516.

MORRIS, M., 1997. Sexual networks and HIV. AIDS, 11 (Sup. A):S209-S216 
NELSON, K. E.; EIUMTRAKUL, S.; CELENTANO, D.; MacLEAN, I.; RONALD, A.; SUPRASERT, S.; HOOVER, D. R.; KUNTOLBRUTA, S. \& ZENILMAN, J. M., 1997. The association of herpes simplex virus type 2 (HSV-2), Haemophilus ducreyi and syphilis with HIV infection in young men in northern Thailand. Journal of Acqui red Immune Deficiency Syndromes and H uman Retrovirology, 16:293-300.

NOPKERSON, T.; MOCK, P. A.; MASTRO, T. D. SANGKHAROMYA, S.; SWEAT, M .; LIMPAKARNJANARAT, K.; LAOSAKKITIBORAN, J.; YOUNG, N. L.; MORSE, S. A.; SCHMID, S. \& WENIGER, B. G., 1998. HIV-1 subtype $E$ incidence and sexually transmitted diseases in a cohort of military conscripts in northern Thailand. Journal of Acquired Immune Deficiency Syndromes and Human Retrovirology, 18:372-379.

O'LEARY, A.; AMBROSE, T. K.; RAFFAELLI, M.; MAIBACH, E.; JEM M OTT, L. S.; JEM M OTT 3rd, J. B.; LABOUVIE, E. \& CELENTANO, D., 1998. Effects of an HIV risk reduction project on sexual risk behavior of low-income STD patients. AIDS Education and Prevention, 10:483-492.

ORGANISTA, K. C.; ORGANISTA, P. B.; GARCIA-DEALBA, J. E.; CASTILLO-MORAN, M. A. \& URETACARRILLO, L. E., 1997. Survey of condom-related beliefs, behaviors, and perceived social norms in Mexican migrant laborers. Journal of Community Health, 22:185-198.

PEERSM AN, G. V. \& LEVY, J. A., 1998. Focus and effectiveness of HIV-prevention efforts for young people. AIDS, 12 (Sup. A):S191-S196.

PERLMAN, D. C.; HENMAN, A. R.; KOCHEMS, L.; PAONE, D.; SALOMON, N. \& DES JARLAIS, D. C. 1999. Doing a shotgun: A drug use practice and its relationship to sexual behaviors and infection risk. Social Science and Medicine, 48:1441-1448.

POPPEN, P. J. \& REISEN, C. A., 1997. Perception of risk and sexual self-protective behavior: A methodological critique. AIDS Education and Prevention, 9:373-390.

REITMAN, D.; St. LAWRENCE, J. S.; JEFFERSON, K. W.; ALLEYNE, E.; BRASFIELD, T. L. \& SHIRLEY, A. 1996. Predictors of African American adolescents' condom use and HIV risk behavior. AIDS Education and Prevention, 8:499-515.

ROMER, D.; BLACK, M.; RICARDO, I.; FEIGELMAN, S.; KALJEE, L.; GALBRAITH, J.; NESBIT, R.; HORNIK, R. C. \& STANTON, B., 1994. Social influences on the sexual behavior of youth at risk for HIV exposure. American Journal of Public Health, 84:977-985.
RUZANY, M. H. \& SZWARCWALD, C. L., 1999. Mortalidade de adolescentes no município do Rio de Janeiro, de 1981 a 1995 - Quantos óbitos poderiam ser evitados? Revista de Pediatria, 75:327-333.

SANTELLI, J. S.; BRENER, N. D.; LOWRY, R.; BHATT, A. \& ZABIN, L. S., 1998. Multiple sexual partners among U.S. adolescents and young adults. Family Planning Perspectives, 30:271-275.

SELLS, C. W. \& BLUM, R. W., 1996. Morbidity and mortality among US adolescents: An overview of data and trends. American Journal of Public Health, 86:513-519.

SINGER, M., 1994. AIDS and the health crisis of the U.S. urban poor: The perspective of critical medical anthropology. Social Science and Medicine, 39:931-948.

SOUZA, J. C. R. P., 1994. Comportamento sexual, DST/ AIDS e uso de drogas entre conscritos do exército brasileiro. Arquivos Brasilei ros de Medicina, 68 : 95-101.

STANTON, B. F.; FITZGERALD, A. M.; LI, X.; SHIPENA H.; RICARDO, I. B.; GALBRAITH, J. S.; TERRERI, N.; STRIJDOM, J.; HANGULA-NDLOVU, V. \& KAHIHUATA, J., 1999. HIV risk behaviors, intentions and perceptions among Namibian youth as assessed by a theory-based questionnaire. AIDS Education and Prevention, 11:132-149.

SZWARCWALD, C. L.; BASTOS, F. I. \& CASTILHO, E. A., 1998. The dynamics of the AIDS epidemic in Brazil: A space-time analysis in the period 19871995. Brazilian Journal of Infectious Diseases, 2: 175-186.

WALLACE, R. \& WALLACE, D., 1995. U.S. apartheid and the spread of AIDS to the suburbs: A multycity analysis of the political economy of spatial epidemic threshold. Social Science and Medicine, 41:333-345

WALTER, H. J. \& VAUGHN, R. D., 1993. AIDS risk reduction among a multiethnic sample of urban high school students. JAMA, 270:725-730.

WARREN, C. W.; SANTELLI, J. S.; EVERETT, S. A.; KANN, L.; COLLINS, J. L.; CASSELL, C.; MORRIS, L.; KOLBE, L. J., 1998. Sexual behavior among U.S. high school students, 1990-1995. Family Planning Perspectives, 30:170-172, 200.

WATERSTON, A., 1997. Anthropological research and the politics of HIV prevention: Towards a critique of policy and priorities in the age of AIDS. Social Science and Medicine, 44:1381-1391. 